\title{
New world Neuropsychiatry
}

\author{
Luis Antonio Pando Orellana* \\ Instituto Politécnico, Mexico
}

*Corresponding author: Luis Antonio Pando Orellana, Escuela Nacional de Ciencias Biológicas, Instituto Politécnico Nacional, México.

To Cite This Article: Luis Antonio Pando Orellana. New world Neuropsychiatry. Am J Biomed Sci \& Res. 2019 - 1(2). AJBSR.MS.ID.000513.

DOI: 10.34297/AJBSR.2019.01.000513

Received: January 18, 2019 | Published: January 22, 2019

\section{Opinion}

The actual curricula of medical schools and hospitals in cerebral cortical and subcortical dysfunctions, demands through the DSM and other instruments such as immunology, infectiology, endocrinology, imageology and the microbiota which constitutes a big percentage of ourselves, at least in the creation of subspecialties in Neuroscience devoted to the notion that Neuropsychiatric pathology is broader than we think.

The relation of our central nervous system with the intestinal nervous system through mainly the vagus, should be studied from macroscopic studies, microscopic and ultrastructure characteristics, functional studies, particularly, the detection of neuropeptides that through blood, blood brain barrier, vagus nerve and lymph reach the central nervous system and modify neurophysiology, and prorogue clinical manifestations of disease mimicking; and broadening the spectra of neuropsychiatric disorders.

Disorders from psychotic to personality changes that we do not know how they work in patients and broaden Neuropsychiatric diagnosis, that are interpreted otherwise and a broaden the spectra of neuropsychiatric issues have to be considered.

If we go through the several "coincidences" of viral, bacterial, fungal and parasite infections through a proper and extended clinical history in patients that experienced a sudden change in the way they handle their circumstance through their immunological history, we encounter several matches not only with HIV, or the so called slow viruses like measles, but common things such as Streptococcal infections group A beta hemolytic or Influenza agents with very, very high IgG that cannot be interpreted as contact if it is thousand times above the reference value in serum searches, and such is the case of Epstein Bar virus or Cytomegalovirus.

Other specialties are trying to understand the several roles that Helicobacter Pylori play in Diabetic problems and metabolically syndromes nowadays. And their changes through the use of antibiotics, that seem to modify conduct as well.

Prions as well and disconnection syndromes that frontalis patients are not always Creutzfeldt- Jacob disease, there has to be minor alterations expressed and not always catastrophic changes (like sclerotizing pan-encephalitis) in Central Nervous System, why? Because they are appearing in our everyday life experience if we search for extended possible pathologies with more curiosity, especially when conductual changes are abrupt and considering that agents of evolution, including mutations in DNA that provoque transcriptase's to change the configuration of proteins from an Alpha structure to Beta and behave like prions.

Economy in biology is a constant in change and There are sequelae of meninge-encephalitis that behave as schizophrenia or severe borderline disorders that with immunotherapy change their course. This is the $10 \%$ rule in every biological phenomenon in a Gauss curve if we go through data bases and can handle values that normalize the curve.

The $20^{\text {th }}$ century was characterized by Freud like interpretation of such phenomena, and that was because they or we did not have proper instruments to answer to mentalists with hard evidence to face the family proposed and social environment hypothesis that mental disease is either genetical, congenital or environmental and that nothing could be done but face them through Word therapy.

That was until the neuro psycho pharmacological industry evolved and medicines that acted through neuro regulation of 4 main neuro regulators being considered: Dopamine, Serotonine, Noreinephrine, GABA (Gamma aminobutyric acid) and just about, and very indirectly through IONS and heavy metals such as Lithium, very toxic by the way and very freely used. Acetylcholine is still an issue for its volatile nanosecond presence. But what about other 
neuropeptides and its manipulation in several diseases, diseases like or cerebral dysfunctions?

Things started to change with antibodies directed to specific areas of the D group in q mapping of chromosome 6 through class II group of antigens in immune-genetical language. (HLA system). Hope for correlation with pathologies such as demielinyzing diseases also epilepsy and schizophrenic spectrum explanation was brought to our attention.

Spectroscopy in magnetic resonance of brain, gave us the possibility of trying to mark associated pathology to anatomy and such was the case of qualicuantitative EEG. Biomarkers started with oncology and now since the past 60 years a very frustrating effort to mark brain damage and identify several neural growth markers.
Efforts to understand Central nervous system have been done particularly in mathematical models of networks since the effort of economists to propose a mind -brain model. Interesting but scarcely useful in therapeutics. The pharmaceutical industry has stalled in a very precarious standby and we researchers with grants.

We have in this violent XXI century to do more with less. We also have to broaden our concept of pandemia with violence and its causes including hunger, hemorrhagic killer diseases, (Arbovirus), interspecies diseases and even migration phenomena. The task is phenomenal and vaccines and also pharmacy seem to be our only poor response with no prevention at all and with less research budgets. Understand the several organ binomial that interact to go for a wider and better explanation other tan Koch postulates or other intended validation systems. The only thing that we cannot do is stop doing it. 